\title{
Composite Fermion Wavefunctions Derived by Conformal Field Theory
}

\author{
Andrea Cappell米 \\ INFN, Via G. Sansone 1, 50019 Sesto Fiorentino - Firenze, Italy
}

(Dated: November 2, 2018)

\begin{abstract}
The Jain theory of hierarchical Hall states is reconsidered in the light of recent analyses that have found exact relations between projected Jain wavefunctions and conformal field theory correlators. We show that the underlying conformal theory is precisely given by the $\mathrm{W}$-infinity minimal models introduced earlier. This theory involves a reduction of the multicomponent Abelian theory that is similar to the projection to the lowest Landau level in the Jain approach. The projection yields quasihole excitations obeying non-Abelian fractional statistics. The analysis closely parallels the bosonic conformal theory description of the Pfaffian and Read-Rezayi states.
\end{abstract}

Introduction. - The search for fractional exchange statistics in quasiparticle excitations of the quantum Hall effect is actively pursued both theoretically [1] 2] and experimentally [3] [4]. The observation of non-Abelian, i.e. multidimensional, statistics, first suggested in the Pfaffian state [5], would be particularly interesting as it could find application to quantum computation [6].

The methods of two-dimensional conformal field theory (CFT) 7] have been extremely useful in this domain. Their description is twofold: they model the dynamics of massless chiral excitations at the edge of the Hall droplet [8], and express analytic wavefunctions for electrons in the lowest Landau level. In particular, the CFT wavefunctions include non-analytic prefactors that make explicit the fractional statistics of excitations. All these methods have been fully developed in the case of Pfaffian and Read-Rezayi [9] states, where they have been instrumental for obtaining several physical results.

The studies of hierarchical Hall states have somehow remained behind, in spite of their experimental relevance stemming from their richness and higher stability. On one side, the Jain theory of the composite fermion [10] predicts very accurate ground-state wavefunctions and has been confirmed by many experiments, but it does not provide full understanding of quasiparticle statistics. On the other side, the CFT description was based on the multicomponent Abelian theory (multicomponent Luttinger liquid) [1]. This approach has had some problems, such as the prediction of several distinct types of electrons.

The precise relation between these two approaches has remained rather unclear till recent results that form the basis of the present work. In a series of papers, Hansson et al 12] have found exact CFT expressions for the Jain wavefunctions projected to the lowest Landau level. These functions have been written in terms of the expected multicomponent Abelian CFT plus some additional requirements to be fully interpreted. Other works [13] have studied the short-distance and pairing (clustering) properties of Jain wavefunctions and compared them to those of the Pfaffian and other non-Abelian Hall states.

In this Letter, we shall relate these result with another theory of the hierarchical Hall states that has been formu- lated independently of the composite fermion picture, by Trugenberger, Zemba and the author [14]. Its main physical input is the incompressibility of the electron fluid and its symmetry under area-preserving diffeomorphisms of the plane, also called W-infinity $\left(W_{1+\infty}\right)$ symmetry. The implementation of this symmetry in the dynamics of edge excitations and the use of results of representation theory lead to a general analysis of conformal theories suitable for spin-polarized Hall states. While they are generally found to be equivalent to the multicomponent Abelian theories, there are special cases with enhanced $S U(n)$ symmetry, where a projection of degrees of freedom is needed to obtain irreducible representations, i.e. elementary excitations. This is achieved through a coset construction [7], as follows:

$$
\widehat{U(1)}^{n} \longrightarrow \widehat{U(1)} \times \widehat{S U(n)}{ }_{1} \longrightarrow \widehat{U(1)} \times \frac{\widehat{S U(n)}}{S U(n)}
$$

The conformal theories obtained in this way were called $W_{1+\infty}$ minimal models [15]; they were found to be in one-to-one correspondence with the Jain states, but to differ from the standard multicomponent Abelian theory owing to the projection of the $S U(n)$ symmetry which, among other things, leads to a unique type of electron and to non-Abelian fusion rules. This projection was also described by introducing a term in the edge Hamiltonian [16]. However, this theory was not yet applied to describe wavefunctions: in this Letter, we complete the analysis and establish a direct relation with the Jain composite fermion theory.

Results. - (i) We show that the Hansson et al. expression of Jain wavefunctions can be completely derived within the $W_{1+\infty}$ minimal models. Furthermore, the projection of the multicomponent Abelian theory realized in the minimal models can be related with the Jain projection to the lowest Landau level.

(ii) We remark that the Jain wavefunctions in the Hansson et al. form are remarkably similar to the Pfaffian and Read-Rezayi wavefunctions expressed in terms of another projection of Abelian theories [17]; the analogies between the two approaches are emphasized.

(iii) We argue that quasiholes excitations over the Jain states should possess non-Abelian fractional statistics as 
predicted by the $W_{1+\infty}$ minimal models; the mechanism for non-Abelian statistics is the same as that of the Pfaffian state and results from the identification of the independent components (effective Landau levels) down to a single one.

Hansson et al. results. - The Jain theory is based on a correspondence between integer, $1 / \nu^{*}=1 / n$, and fractional, $1 / \nu=p+1 / n$, Hall effects ( $p$ even); this is realized by introducing the ground state wave function [10]:

$$
\Psi_{\nu}=\mathcal{P}_{L L L} \prod_{i<j}^{N}\left(z_{i}-z_{j}\right)^{p} \Psi_{\nu^{*}=n} .
$$

The term $\Psi_{\nu^{*}=n}$ is the Slater determinant for $N$ electrons completely filling the first $n$ Landau levels, say putting $N / n$ of them in each level; $\mathcal{P}_{L L L}$ expresses the projection to the lowest Landau level. Note that electrons originally placed in the $j$-th level, $j=1,2, \ldots$, have angular momentum of one-particle states shifted by $(1-j)$.

In their careful study of quasi-particle excitations over the Laughlin and other states, Hansson et al. 12] obtained an exact rewriting of (2). Using non-trivial determinant identities, they found the following formula:

$$
\Psi_{\nu}=\mathcal{A}\left[\prod_{i<j}^{N / 2} w_{i j}^{p+1} \prod_{k=1}^{N / 2} \partial_{z_{k}} \prod_{i<j}^{N / 2} z_{i j}^{p+1} \prod_{i, j}^{N / 2}\left(z_{i}-w_{j}\right)^{p}\right] .
$$

In this expression, we considered the $n=2$ case for simplicity $(\nu=2 /(2 p+1))$, and put $N_{1}=N_{2}=N / 2$ electrons in the first and second level, respectively (the result can be extended to $N_{1}>N_{2}$ and also to $n>2[12]$ ). Electron coordinates in the first and second level were denoted by $w_{i}$ and $z_{j}, i, j=1, \ldots, N / 2$, respectively, and their differences by $w_{i j}=w_{i}-w_{j}$ and $z_{i j}=z_{i}-z_{j}$. The symbol $\mathcal{A}$ indicates antisymmetrization with respect to all $N$ electron coordinates.

The remarkable expression (3) admits a simple description in terms of CFT correlators. From the Jastrow factors inside square brakets, one can recognize the wavefunction of the standard two-component Abelian CFT description of hierarchical states [11] with so-called $K$ matrix equal to $\left(\begin{array}{cc}p+1 & p \\ p & p+1\end{array}\right)$; this corresponds to the following correlator of two kinds of conformal fields (vertex operators):

$$
\Psi_{\nu}=\mathcal{A}\left[\left\langle\left(\partial_{z_{1}} V_{+}\right) \cdots\left(\partial_{z_{N / 2}} V_{+}\right) V_{-} \cdots V_{-}\right\rangle\right] .
$$

The vertex operators can be written in terms of charged $\phi$ and neutral $\varphi$ scalar fields as $V_{ \pm}=e^{i \sqrt{p+\frac{1}{2}} \phi} e^{ \pm i \frac{1}{\sqrt{2}} \varphi}$ (see 15] for details).

The expressions (3) and (4) can be interpreted as describing electrons belonging to the first two Landau levels, with the derivatives realizing the shift of angular momentum for the second level and the antisymmetrization ensuring identical electrons.
Analogies with the Pfaffian state. - Hansson et al. expression (3) is very similar to the Pfaffian state in the two-component Abelian CFT description developed in 17], which reads $(1 / \nu=M+1, M$ odd):

$$
\Psi_{\text {Pfaff }}=\mathcal{A}\left[\prod_{i<j}^{N / 2} w_{i j}^{M+2} \prod_{i<j}^{N / 2} z_{i j}^{M+2} \prod_{i, j}^{N / 2}\left(z_{i}-w_{j}\right)^{M}\right]
$$

corresponding to the matrix $K=\left(\begin{array}{cc}M+2 & M \\ M & M+2\end{array}\right)$. After antisymmetrization, the expression (5) can be shown to be identical to the standard form $\Psi_{\text {Pfaff }}=$ $\prod z_{i j}^{M+1} \operatorname{Pf}\left(1 / z_{i j}\right)[5]$.

The explanation for such Abelian representation of the Pfaffian state rests in the physics of electron pairing. Before antisymmetrization, the state (5) describes distinguishable electrons, possessing a twofold quantum number, say isospin. Their wave function does not vanish (for $M=0$ ) when two electrons with different isospin meet at the same point $z_{1}=z_{2}=z$, indicating their pairing; when a third electron approaches, it necessarily vanish as $\Psi \sim\left(z_{3}-z\right)^{2}$. After (anti)symmetrization, all electrons become identical, i.e. the theory only contains isospin singlets, but the pairing property is retained, that is characteristic of the Pfaffian state.

The Abelian representation of the Pfaffian extends easily to quasihole excitations [17]: before projection, these are of two kinds, each one coupling to its electron: after projection, they become identical and yield a multidimensional representation of the braid group, i.e. to nonAbelian statistics [18]. This is a nice way to understand non-Abelian statistics within the standard Abelian setting: it is just a consequence of the projection to identical electrons.

The similarity of the expressions (3) and (5) for the Jain and Pfaffian states brings in a series of results that will be relevant for the following discussion. A first observation is that the Jain states possess the same vanishing behaviour of the Pfaffian and Read-Rezayi states. When three or more particles approach the same point, Eq. (3) behaves as:

$$
\begin{array}{ll}
\Psi_{n=2} \sim z_{12}^{p-1}\left(z_{13}^{p+1} z_{14}^{p+1} \cdots\right), & \frac{1}{\nu}=p+\frac{1}{2},(6) \\
\Psi_{n=3} \sim\left(z_{12} z_{13} z_{23}\right)^{p-1}\left(z_{14} z_{15} \cdots\right)^{p+1}, \frac{1}{\nu}=p+\frac{1}{3} .(7)
\end{array}
$$

In the second expression, we also reported the $n=3$ case to be compared with the $\mathbb{Z}_{3}$ parafermionic Read-Rezayi state [9] 17]. The result (6) can be easily proven by using a graphical representation for the action of derivatives in the expression (3) [19].

Therefore, we see that the $M=0$ Pfaffian and the $p=1$ Jain states have the same pairing properties. The difference between them is that the Pfaffian (ReadRezayi) state is the lowest order polynomial, i.e. lowest angular momentum state, obeying that type of pairing (clustering), while the Jain state has higher angular 


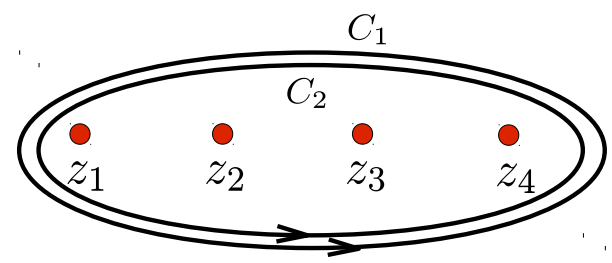

FIG. 1. The contours for the screening charges.

momentum. Another way to put this fact is that the Pfaffian is the lowest momentum zero-energy state of a three-body short-distance potential, while Jain state is an excited state. A short-distance potential that could similarly single out the Jain state has not been found [13].

Derivation of Jain wavefunctions. - We start from the two-component Abelian theory and describe the projection of the $S U(2)$ symmetry (1) on wavefunctions (see [16] for an introduction). The neutral parts of the vertex operators $V_{ \pm}$in (4) have associated isospins values $t_{z}= \pm 1 / 2$ that should be identified; the resulting field is only characterized by its Virasoro dimension $h=t^{2} / 2$, according to the coset projection (11):

$$
\widehat{U(1)} \times \frac{\widehat{S U(2)}}{S U(2)} \sim \widehat{U(1)} \times \operatorname{Vir} .
$$

A convenient way to perform the projection is by using the Dotsenko-Fateev screening operators $Q_{ \pm}$[7]. These have vanishing scale dimension but non-vanishing isospin $t_{z}= \pm 1$, and can relate the two electron fields:

$$
V_{-} \sim V_{+}=Q_{+} V_{-}, \quad Q_{+}=J_{0}^{+}=\oint d u J^{+}(u) .
$$

Note that in this theory the screening operators are the zero modes of the $\widehat{S U(2)}$ affine algebra. Using a standard procedure, the correlator of, say, four electrons can be expressed in terms of four vertex operators and two screening charges, needed for achieving zero total isospin, as follows:

$$
\Psi=\left\langle\oint_{C_{1}} J^{+} \oint_{C_{2}} J^{+} V_{-}\left(z_{1}\right) V_{-}\left(z_{2}\right) V_{-}\left(z_{3}\right) V_{-}\left(z_{4}\right)\right\rangle .
$$

The next step is to choose the contours $C_{1}, C_{2}$. In principle, there are several choices, corresponding to different intermediate states in the fusion of two electrons. Actually, after projection the fields in the Virasoro theory acquire non-Abelian fusion rules, corresponding to the addition of isospins, $\{1 / 2\} \times\{1 / 2\}=\{0\}+\{1\}$ (these are nothing else than the the $c \rightarrow 1$ limit of the fusion rules of minimal Virasoro models).

The ground state wavefunction should be completely (anti)symmetric with respect to all electrons; this requires the contours to encircle all of them as shown in
Fig. 1. Unfortunately, this choice yields a vanishing result (explicitly checked in [16]), because the contours can be deformed at infinity where they correspond to acting on the invariant right vacuum, $\langle 0| J_{0}^{-}=0$.

The solution of this puzzle is to identify the two electron fields through another $\widehat{S U(2)}$, generator with same $t_{z}=1$ isospin but leading to non-vanishing results. The natural replacement is,

$$
J_{0}^{+} \rightarrow J_{-1}^{+} .
$$

Acting on vertex operators, $J_{-1}^{+}$yields the first descendent in the tower of states of the affine representation,

$$
J_{-1}^{+} V_{-} \sim \partial_{z} V_{+},
$$

as easily checked by using the vertex operator algebra. Note that the operators $\left\{J_{-1}^{+}, J_{1}^{-}, 1 / 2-J_{0}^{3}\right\}$ form another $S U(2)$ algebra contained in the affine algebra $\widehat{S U(2)}{ }_{1}[7]$, that is equivalent for realizing the projection (8).

Thus, we consider the following expression for the four electron wavefunction:

$$
\Psi^{\prime}=\left\langle J_{-1}^{+} V_{-}\left(z_{1}\right) J_{-1}^{+} V_{-}\left(z_{2}\right) V_{-}\left(z_{3}\right) V_{-}\left(z_{4}\right)\right\rangle+\text { perm }
$$

This is actually equal to the Hansson et al. expression (41), owing to (12).

Therefore, we have obtained a derivation of the Jain composite fermion wavefunction entirely from symmetry arguments, through the construction of the $W_{1+\infty}$ minimal models and their application to describe wavefunctions. This results is rewarding insofar as it supports the universality and robustness of the Jain theory; furthermore, it can be used to obtain a consistent description of excitations, as discussed in the next subsection.

Let us remark that Eq.(12) strictly holds for the neutral part of the vertex operators $V_{ \pm}$: for the derivative to act on the whole electron field, as in (4), one needs to deform the $S U(2)$ generator for including a charged $\widehat{U(1)}$ part: $J_{-1}^{+} \rightarrow J_{-1}^{+}+\sqrt{2} \alpha_{0} \alpha_{-1}$. Upon varying the relative coefficient, it also possible to obtain a $p$-dependent family of wave functions, the Jain wavefunction being one of them. This ambiguity could be fixed by other physical requirements, but the pairing (6) and fractional statistics properties are independent of it. Note also that the $W_{1+\infty}$ derivation of Jain wavefunctions extends to more than two Landau levels [19]: for $n=3$, two $S U(3)$ generators $J_{-1}^{\alpha_{i}}$ are employed, whose action on vertex operators leads to single and double derivatives in agreement with Hansson et al. results [12].

Quasihole excitations and non-Abelian statistics.Here we shall be rather brief for lack of space, and defer to [19] for a complete discussion. The analysis is similar to that of the Pfaffian state [18] in its Abelian description 17]. In the two-component Abelian theory of Jain states before projection, there are two quasihole excitations of smallest charge with isospins $t_{z}= \pm 1 / 2$ [16]. They are 
represented by fields $H_{ \pm}$that couple to the respective electron fields $V_{ \pm}$, owing to the fusion rules $H_{ \pm} V_{\mp} \sim I$. In the Jain description, each quasihole is made in one effective Landau level, and Hansson et al. have verified that the insertion of $H_{ \pm}$in the CFT correlator (41) reproduces the corresponding Jain wavefunction [12].

After the projection, the two quasiholes should also be identified, $H_{+} \sim J_{0}^{+} H_{-}$; moreover, they should occur in even number with vanishing total isospin. For four quasiholes, there are three possible amplitudes that are associated to the same physical excitation; the first is:

$$
\begin{aligned}
\Psi_{(12,34)}=\mathcal{A}_{z_{i}}\langle & {\left[H_{+}\left(\eta_{1}\right) H_{+}\left(\eta_{2}\right) H_{-}\left(\eta_{3}\right) H_{-}\left(\eta_{4}\right)\right.} \\
& \left.+(+\leftrightarrow-)] \prod V_{e}\left(z_{i}\right)\right\rangle,
\end{aligned}
$$

where we collectively denoted by $V_{e}$ the two electron fields. The other two amplitudes, $\Psi_{(13,24)}$ and $\Psi_{(14,24)}$, amount to different assignments of $( \pm)$. These three amplitudes give rise to a multidimensional representation of the braid group $\mathcal{B}_{4}$, i.e. to non-Abelian statistics.

Therefore, the description of Jain quasiholes within the $W_{1+\infty}$ minimal models naturally implies their nonAbelian fractional statistics. This is our second main result.

Inspection of the $2 k$-hole states for $k=2,3$ shows that there are no degeneracies among the possible amplitudes, in contrast with the Pfaffian case [18]; thus, their number grows like $d_{k}=2 k ! / 2(k !)^{2} \sim 2^{2 k} / \sqrt{k}$. The same multiplicities can be inferred by tensoring isospin onehalf representations as predicted by CFT.

The Jain theory also considers quasiholes made in a single effective level, involving one type of field only, say $H_{+}$. These excitations obey Abelian fractional statistics, and are not allowed in the $W_{1+\infty}$ minimal models because they violate the condition of vanishing total isospin. Their actual relevance depends on the size of their gap, not predicted by the CFT approach.

We remark that the results presented here do not yet provide a proof of non-Abelian statistics in Jain states, lacking an understanding of the energetics of excitations. In the case of the Pfaffian state [9], the three-body short-distance electron potential identifies the exact zeroenergy subspace in which the non-Abelian excitations have the desired degeneracy. Hopefully, corresponding steps in the hierarchical states could be made by developing the studies in [13], including numerical analyses of the projection [20] and entanglement entropy [21], and complete the arguments presented here. Present experiments testing non-Abelian statistics [1] [4] could also be tried on Jain states, if higher magnetic fields $(\nu<2)$ can be attained.

We are indebted to G. R. Zemba for collaboration in the early stages of this work. We also thank A. Bernevig, M. C. Diamantini, T.H. Hansson, N. Regnault, I. D. Rodriguez, K. Schoutens, C. A. Trugenberger and S. Viefers for interesting discussions. The Galileo Galilei Institute for Theoretical Physics, Florence, and Nordita, Stockholm, are acknowledged for their kind hospitality.

* andrea.cappelli@fi.infn.it

[1] P. Bonderson, A. Kitaev, K. Shtengel, Phys. Rev. Lett. 96, 016803 (2006); W. Bishara, P. Bonderson, C. Nayak, K. Shtengel, J. K. Slingerland, Phys. Rev. B 80, 155303 (2009); B. I. Halperin, A. Stern, I. Neder, B. Rosenow, Phys. Rev B 83, 155440 (2011).

[2] N. R. Cooper, A. Stern, Phys. Rev. Lett. 102, 176807 (2009); K. Yang, B. I. Halperin, Phys. Rev. B 79, 115317 (2009).

[3] D. T. McClure, W. Chang, C. M. Marcus, L. N. Pfeiffer, K. W. West, preprint arXiv:1112.0538 Phys. Rev. Lett. in press; R.L. Willett, L.N. Pfeiffer, K.W. West, preprint arXiv:1204.1993 S. An, P. Jiang, H. Choi, W. Kang, S. H. Simon, L. N. Pfeiffer, K. W. West, K. W. Baldwin, preprint arXiv:1112.3400.

[4] W. E. Chickering, J. P. Eisenstein, L. N. Pfeiffer, K. W. West, Phys. Rev. B 81, 245319 (2010).

[5] G. Moore, N. Read, Nucl. Phys. B 360, 362 (1991).

[6] C. Nayak, S. H. Simon, A. Stern, M. Freedman, S. Das Sarma, Rev. Mod. Phys. 80, 1083 (2008).

[7] P. Di Francesco, P. Mathieu, D. Senechal, Conformal Field Theory, Springer (1999), Berlin.

[8] X. G. Wen, Quantum Field Theory of Many-body Systems, Oxford Univ. Press (2007), Oxford.

[9] N. Read, E. Rezayi, Phys. Rev. B 59, 8084 (1999).

[10] J. K. Jain, R. K. Kamilla, Int. J. Mod. Phys. B 11, 2621 (1997); J. K. Jain, Composite Fermions, Cambridge Univ. Press (2007), Cambridge.

[11] N. Read, Phys. Rev. Lett. 65, 1502 (1990); J. Fröhlich, A. Zee, Nucl. Phys. B 364, 517 (1991); X.-G. Wen, A. Zee, Phys. Rev. B 46, 2290 (1992).

[12] T.H. Hansson, C.-C. Chang, J.K. Jain, S. Viefers, Phys. Rev. Lett. 98, 076801 (2007), Phys. Rev. B 76, 075347 (2007); J. Suorsa, S. Viefers, T. H. Hansson, New J. Phys. 13, 075006 (2011).

[13] S. H. Simon, E. H. Rezayi, N. R. Cooper Phys. Rev. B 75, 075318 (2007); B. A. Bernevig, F. D. M. Haldane, Phys. Rev. Lett. 102, 066802 (2009); N. Regnault, B. A. Bernevig, F. D. M. Haldane, Phys. Rev. Lett. 103, 016801 (2009); N. Moran, A. Sterdyniak, I. Vidanovic, N. Regnault, M.V. Milovanovic, preprint arXiv:1205.2388.

[14] A. Cappelli, C.A. Trugenberger, G.R. Zemba, Nucl. Phys. B 396, 465 (1993), Phys. Rev. Lett. 72, 1902 (1994).

[15] A. Cappelli, C.A. Trugenberger, G.R. Zemba, Nucl. Phys. B 448, 470 (1995).

[16] A. Cappelli, G.R. Zemba, Nucl. Phys. B 540, 610 (1999).

[17] A. Cappelli, L. S. Georgiev, I. T. Todorov, Nucl. Phys. B 599, 499 (2001).

[18] C. Nayak, F. Wilczek, Nucl. Phys. B 479, 529 (1996).

[19] A. Cappelli, G. R. Zemba, in preparation.

[20] A. Cappelli, C. Mendez, J. M. Simonin, G. R. Zemba, Phys. Rev. B 58, 16291 (1998).

[21] I. D. Rodriguez, S. H. Simon, J. K. Slingerland, preprint arXiv:1111.3634 